\title{
Adjunctive Lisdexamfetamine Dimesylate Therapy in Adult Outpatients With Predominant Negative Symptoms of Schizophrenia: Open-Label and Randomized-Withdrawal Phases
}

\author{
Robert A Lasser*,', Bryan Dirks², Henry Nasrallah ${ }^{3}$, Courtney Kirsch ${ }^{2}$, Joseph Gao ${ }^{2}$, Michael L Pucci ${ }^{4}$, \\ Mary A Knesevich ${ }^{5}$ and Jean-Pierre Lindenmayer ${ }^{6}$ \\ 'Formerly of Shire Development LLC, Wayne, PA, USA; ${ }^{2}$ Shire Development LLC, Wayne, PA, USA; ${ }^{3}$ University of Cincinnati College of Medicine, \\ Cincinnati, OH, USA; ${ }^{4} \mathrm{SCl}$ Scientific Communications \& Information, Parsippany, NJ, USA; ${ }^{5}$ University Hills Clinical Research, Irving, TX, USA; \\ ${ }^{6}$ New York University School of Medicine, New York, NY, USA
}

Negative symptoms of schizophrenia (NSS), related to hypodopaminergic activity in the mesocortical pathway and prefrontal cortex, are predictive of poor outcomes and have no effective treatment. Use of dopamine-enhancing drugs (eg, psychostimulants) has been limited by potential adverse effects. This multicenter study examined lisdexamfetamine dimesylate (LDX), a d-amphetamine prodrug, as adjunctive therapy to antipsychotics in adults with clinically stable schizophrenia and predominant NSS. Outpatients with stable schizophrenia, predominant NSS, limited positive symptoms, and maintained on stable atypical antipsychotic therapy underwent a 3-week screening, I0-week open-label adjunctive LDX (20-70 mg/day), and 4-week, double-blind, randomized, placebo-controlled withdrawal. Efficacy measures included a modified Scale for the Assessment of Negative Symptoms (SANS-18) and Positive and Negative Syndrome Scale (PANSS) total and subscale scores. Ninety-two participants received open-label LDX; 69 received doubleblind therapy with placebo $(n=35)$ or LDX $(n=34)$. At week 10 (last observation carried forward; last open-label visit), mean (95\% confidence interval) change in SANS-18 scores was $-12.9(-15.0,-10.8 ; P<0.0001)$. At week $10,52.9 \%$ of participants demonstrated a minimum of $20 \%$ reduction from baseline in SANS-I 8 score. Open-label LDX was also associated with significant improvement in PANSS total and subscale scores. During the double-blind/randomized-withdrawal phase, no significant differences (change from randomization baseline) were found between placebo and LDX in SANS-18 or PANSS subscale scores. In adults with clinically stable schizophrenia, open-label LDX appeared to be associated with significant improvements in negative symptoms without positive symptom worsening. Abrupt LDX discontinuation was not associated with positive or negative symptom worsening. Confirmation with larger controlled trials is warranted.

Neuropsychopharmacology (2013) 38, 2I40-2 I49; doi:10.1038/npp.20 I3.1 I I; published online 12 June 2013

Keywords: lisdexamfetamine dimesylate; adjunctive; negative symptoms; schizophrenia

\section{INTRODUCTION}

A substantial segment of patients with schizophrenia demonstrates persistent negative symptoms of schizophrenia (NSS): blunted affect, alogia, asociality, avolition/ amotivation, anergia, and anhedonia (Foussias and Remington, 2010; Stahl and Buckley, 2007). Negative symptoms are predictive of poor outcomes and associated with impaired psychosocial functioning, decreased cogni-

*Correspondence: Dr RA Lasser, Department of Neuroscience, inVentiv Health Clinical, 1787 Sentry Parkway West, Suite 300, Building 16, Blue Bell, PA 19422, USA, Tel: +1 215 591 8383, Fax: + | 609514 2165, E-mail: robert.lasser@inventivhealth.com Received 15 January 2013; revised 9 April 2013; accepted 27 April 2013; accepted article preview online 8 May 2013 tive performance, reduced quality of life, and deficits in social, recreational, and work abilities (Milev et al, 2005). While hyperactivity of dopamine transmission via $\mathrm{D}_{2}$-receptors in subcortical regions, including the mesolimbic pathway and nucleus accumbens, is believed to contribute to positive symptoms (Toda and Abi-Dargham, 2007), deficits in transmission at $D_{1}$-receptors, particularly in the mesocortical pathway and prefrontal cortex, may contribute to negative symptoms and cognitive impairments (Guillin et al, 2007).

Antipsychotic agents have very limited effectiveness in reducing persistent NSS; none demonstrate consistent, clinically significant improvements (Buckley and Stahl, 2007). The unmet need for effective NSS treatments is well recognized (Kirkpatrick et al, 2006; Laughren and Levin, 2011). Given the proposed role of dopamine hypoactivity, 
dopamine-enhancing compounds that directly or indirectly activate $\mathrm{D}_{1}$-receptors have been hypothesized to improve NSS (Tamminga, 2006). Early case series and open-label studies of amphetamines suggested the potential for improvement in NSS using dopamine-enhancing drugs (Angrist et al, 1982; Cesarec and Nyman, 1985; Desai et al, 1984). Subsequently, such pharmacological interventions were largely abandoned because of their potential psychotogenic effects (Laruelle et al, 2005). The occurrence of psychotogenic effects, however, following administration of dopamine-enhancing drugs, varies greatly across studies. These effects may be moderated by a number of factors including the patient's clinical state (acute $v s$ chronic), antipsychotic treatment status (drug-free $v s$ effective antipsychotic medication treatment), symptomatic presentation (predominantly positive $v s$ predominantly negative symptoms), and type of psychostimulant administered (Curran et al, 2004; Lieberman et al, 1987). Taking advantage of such attenuating factors provides the rationale for use of adjunctive psychostimulant treatment of patients with predominant NSS.

Lisdexamfetamine dimesylate (LDX), a prodrug of d-amphetamine, is approved for treatment of attentiondeficit/hyperactivity disorder (ADHD) in both children and adults (Vyvanse package insert, 2012). After oral ingestion, LDX is converted to l-lysine and active d-amphetamine. Given the effects of d-amphetamine on dopamine transmission, we hypothesized a potential therapeutic role for LDX in NSS treatment. The present pilot study was designed to explore the efficacy and safety of LDX as an adjunctive therapy to a stable dose of atypical antipsychotic medication in adults with clinically stable schizophrenia and predominant negative symptoms.

\section{PARTICIPANTS AND METHODS}

This multicenter, open-label treatment and double-blind placebo-controlled randomized-withdrawal study of LDX enrolled outpatients at 28 US sites from September 2009 to January 2011. In recognition of the potential for d-amphetamine to exacerbate psychosis, as noted in the Introduction, the study incorporated features that are described in detail here in the Methods section to ensure the safe administration of LDX. Briefly, these included careful selection of participants who were in a stable phase of illness with low levels of positive symptoms and who could be monitored by a close informant outside the clinic; an open-label treatment phase to ensure that any potential emerging safety concerns would be promptly recognized and addressed; and a randomized-withdrawal phase to assess any safety concerns with abrupt withdrawal of amphetamine treatment. The 4-week randomizedwithdrawal phase was also designed to serve as a pilot assessment of an efficacy signal for planning future large, controlled clinical trials. The study protocol was conducted in accordance with current applicable regulations, International Conference on Harmonization, Good Clinical Practice guidelines, and local ethical/legal requirements. Signed, informed consent was required from participants or their legally authorized representative after complete explanation of the study and before entry. Approval from the institutional review board/independent ethics committee at study sites was obtained.

\section{Participants}

The study was restricted to outpatients (18-55 years) with clinically stable Diagnostic and Statistical Manual of Mental Disorders, Fourth Edition, Text Revision (American Psychiatric Association, 2000) schizophrenia, minimal positive symptoms, and predominant NSS who adhered to an established treatment with antipsychotic medication and had a stable relationship with an acceptable informant throughout the study. Participants had a primary diagnosis of schizophrenia for $\geqslant 2$ years before screening and a score $\geqslant 55$ on the Scale for the Assessment of Negative Symptoms (Andreasen, 1984) (SANS) sum of items: 1-6, 8-12, 14-16, and 18-21 (SANS-18). At least moderate scores $(\geqslant 3)$ on $\geqslant 2$ SANS global items (affective flattening, alogia, avolitionapathy, or anhedonia-asociality) were required. Only clinically stable participants in the nonacute phase of illness for $\geqslant 12$ weeks could enroll; maintenance on monotherapy with long-acting risperidone injection or oral risperidone, paliperidone, quetiapine, aripiprazole, or olanzapine for $\geqslant 12$ weeks with no dose change $>10 \%$ within 1 month of screening was required.

Exclusion criteria were intended to prevent enrollment of participants with notable positive symptoms or at greatest risk of adverse events (AEs). Participants with Positive and Negative Syndrome Scale (Kay et al, 1987) (PANSS)-positive subscale score $\geqslant 20$ or score $\geqslant 4$ on positive subscale items for delusions (P1), hallucinatory behavior (P3), or suspiciousness/persecution (P6) were excluded. To minimize possible confounds of negative symptoms with depressive and bradykinesia symptoms, participants with baseline score $\geqslant 9$ on the Calgary Depression Scale for Schizophrenia (Addington et al, 1990) (CDSS) at baseline or Simpson Angus Scale (Simpson and Angus, 1970) (SAS) akinesia score (items $1-7) \geqslant 2$ were excluded.

Participants considered treatment-refractory were excluded as were those considered at-risk for suicide. Owing to concerns of potential prolonged QTc interval, patients on ziprasidone were excluded from the trial (Geodon package insert, 2010). Other key exclusion criteria included history of ADHD or seizures, cardiovascular disease that may increase vulnerability to sympathomimetic effects of LDX, recent ( $<6$ months) history of substance abuse or dependence (excluding nicotine), and use of psychotropic drugs other than permitted antipsychotic medication.

\section{Study Design}

For retrospective and prospective confirmation of clinical stability and predominant NSS, the study included a 3-week screening phase with weekly visits. Throughout screening, inclusion and exclusion criteria were reviewed, including assessment of positive and negative symptomatology via PANSS and SANS rating scales. Participants continuing to meet entry criteria entered a 10 -week open-label phase comprising 7-week, adjunctive LDX dose-optimization and 3 -week dose-maintenance periods, each with weekly visits. Participants initiated therapy at $20 \mathrm{mg} /$ day LDX for 2 weeks. Thereafter, LDX could be increased in $10 \mathrm{mg} /$ week increments 
(up to $70 \mathrm{mg} /$ day) based on investigator's assessment of the participant's overall clinical condition. For reference, LDX $20 \mathrm{mg}$ and $70 \mathrm{mg}$ capsules contain d-amphetamine content of 5.9 and $20.8 \mathrm{mg}$, respectively. LDX was administered upon awakening; antipsychotic medications remained unchanged from screening. During dose optimization and maintenance, one 10 -mg dose reduction was permitted. Participants were maintained at optimized dose during the 3-week, dose-maintenance period.

Participants exhibiting any reduction in week-10 SANS-18 score and without changes in physical examination, vital signs, laboratory assessments, or electrocardiograms (ECGs), that would preclude further LDX treatment, were eligible to participate in the 4-week double-blind/randomized-withdrawal phase. Suitability to continue the study was assessed at all visits. Along with investigator's clinical judgment, prespecified discontinuation criteria included $\geqslant 25 \%$ increase in PANSS total score, $\geqslant 2$-point increase from baseline on PANSS-positive items assessing delusions (P1), hallucinatory behavior (P3), or suspiciousness/persecution (P6) at two consecutive visits, or increase in suicidal ideation or nonsuicidal, self-injurious behavior. Participants meeting randomization criteria at week 10 were allocated to placebo (abrupt discontinuation of adjunctive LDX) or continued optimal dose of LDX in a 1:1 ratio by computer-generated randomization schedule. An interactive voice-/web-response system was used for randomization assignment, and to dispense and manage LDX or matching placebo. Randomization was stratified by response status, with responders defined as participants with $\geqslant 20 \%$ reduction in SANS-18 score and partial responders with a reduction $<20 \%$ but $>0 \%$. Dose adjustments were prohibited during the double-blind/randomized-withdrawal phase, which ended at week 14 or at an early termination (ET) visit. Double-blind treatment was provided as overencapsulated, indistinguishable capsules of LDX or matching placebo.

\section{Efficacy and Safety Measures}

At all study visits, negative symptoms were assessed with the SANS. The SANS-18 score (primary efficacy variable) was the sum of all nonglobal item ratings of affective flattening, inappropriate affect, alogia, avolition/apathy, and anhedonia/asociality. Items are scored from 0 (not at all) to 5 (severe). This modified SANS score excluded attention domain and global items to reduce potential bias in favor of LDX related to its known effects on attention and to focus on individual core negative symptom items. A cutoff of $\geqslant 55$ on the SANS-18 total score for inclusion ensured a population with moderate-to-severe NSS. This score is comparable to an average score of 3 across all SANS-18 items. A SANS-25 total score of 75 is also comparable to an average score of 3 (eg, moderate severity) across all items (Storosum et al, 2002). Although the SANS is a widely accepted, validated standard measure of treatment outcome, additional psychometric testing has not validated the SANS18. The full SANS scale (SANS-25) was administered at all visits. The SANS-25 total score and modified SANS-18 total score were calculated for all visits.

The PANSS was completed at all visits to assess safety (potential emergence of positive symptoms) and efficacy (impact of treatment on negative symptoms). Trained raters, assessing the SANS and PANSS at multiple visits, were blinded to study protocol and hypothesis, and visit status.

AEs, collected at all visits, were coded using the Medical Dictionary for Regulatory Activities Version 11.1. Events starting or worsening on or after the date of the first dose and no later than 3 days following the last dose of study medication were treatment-emergent (TEAEs). AEs were categorized as being from the open-label or double-blind/ randomized-withdrawal phase based on start date. Systolic (SBP) and diastolic (DBP) blood pressure were assessed at all study visits. Additionally, 12-lead ECGs were performed at screening, baseline, randomization (week 10), and study end (week 14/ET).

Additional safety measures assessed effects of LDX on depressive and EPS. The CDSS, a 9-item measure of depressive symptoms in people with schizophrenia, was administered throughout the screening period, at baseline, and weeks 3, 7, 10, and 14/ET. The 10-item SAS measured EPS. Other assessments of EPS included Barnes Akathisia Scale (BAS), (1989), a measure of akathisia associated with antipsychotic medication, and Abnormal Involuntary Movement Scale (Guy, 1976) (AIMS). EPS measures were assessed at weeks 0, 10, and 14/ET. The Amphetamine Cessation Symptom Assessment (McGregor et al, 2008) (ACSA), a self-reported scale of withdrawal symptoms (16 symptoms rated on a 5 -point scale ( $[0=$ not at all to $4=$ extremely]) was administered at baseline and weeks 10-14/ET). Compliance with study treatment was monitored using medication return and drug-accountability logs; participants taking $80-120 \%$ of study treatments were reported as compliant.

\section{Statistical Analysis}

Because of the exploratory nature of the primary assessment in the open-label phase, formal power calculation was not performed. Target sample size was based on power calculations for the double-blind/randomized-withdrawal phase. To detect an effect size of 0.6 for LDX ( $v$ s placebo) on change in SANS-18 score from randomization baseline to double-blind/randomized-withdrawal phase endpoint with a two-sided two-sample $t$-test at a significance level of $5 \%$ and a power of $60 \%$, a sample size of 29 per group was needed. In a study that assessed the effects of treatment on NSS in patients with chronic schizophrenia and predominant NSS, response ( $\geqslant 20 \%$ decrease in symptom scores) rates with ziprasidone or amisulpride, respectively, were 56 and $62 \%$ in the intention-to-treat populations and 62 and $74 \%$ in evaluable participants (Olie et al, 2006). Assuming similar response, 66 enrollees would be responders or partial responders (any reduction in SANS-18 score) at week 10 and have available efficacy data after randomization. So, target enrollment was 88 adults.

Open-label safety and efficacy data were assessed in the safety analysis set (all enrolled participants who took $\geqslant 1$ dose of LDX and had one postbaseline safety assessment) and full analysis set (FAS, all enrolled participants who took $\geqslant 1$ dose of LDX and had one postbaseline efficacy assessment). Effects of LDX (or placebo) in the doubleblind/randomized-withdrawal phase were assessed in the randomized safety analysis set and randomized FAS 
(participants who took $\geqslant 1$ dose of randomized treatment and had $\geqslant 1$ safety/efficacy measure in the double-blind phase). Efficacy data from the double-blind/randomized-withdrawal phase were also evaluated in the randomized evaluable set (RES, all randomized participants who were responders at week 10 and had SANS-18 total scores at week 14/ET).

Change in SANS-18 score from baseline to open-label endpoint was the primary efficacy endpoint. The SANS-18 score was summarized by visit for the FAS, using both the last observation carried forward (LOCF) approach and observed cases. A two-sided paired $t$-test was performed for change at each visit. Changes in SANS-18 scores were also validated in the double-blind/randomized-withdrawal phase (RES and randomized FAS) using analysis of covariance model with treatment group as a factor, and randomization baseline SANS-18 score as a covariate. Similar analyses were employed for secondary efficacy measures. In a post hoc analysis, change in SANS-18 was also assessed using a mixed-effects model for repeated measures (MMRM) including fixed factors for visit, a covariate of open-label baseline, and the interaction of the baseline covariate and visit. To examine the potential for differential site effects, change in SANS-18 total score was also compared post hoc for sites with high $(>10)$ vs low $(\leq 10)$ participant enrollment. The proportion of treatment responders was descriptively summarized during the open-label phase. The proportion of participants meeting response criteria during the double-blind/randomized-withdrawal phase was compared between treatment groups using the Fisher's Exact Test for the RES and Cochran-Mantel-Haenszel test for the randomized FAS. Throughout the study, safety assessments were descriptively summarized. No adjustment for multiple comparisons was made in this study.

\section{RESULTS}

Enrollment and disposition of participants are illustrated in Figure 1. Overall, $23(25.0 \%)$ participants did not complete the 10-week open-label phase, including 5 (5.4\%) who met prespecified withdrawal criteria. During the double-blind/ randomized-withdrawal phase, 13 participants (18.8\%) withdrew, including $2(2.9 \%), 1$ from each group, who met prespecified withdrawal criteria. At the beginning of the dose-maintenance period (week 7), median daily LDX dose was $50 \mathrm{mg} ; 65.8 \%$ of participants were receiving $\geqslant 50 \mathrm{mg} /$ day. Overall, $93.5 \%$ of participants met criteria for compliance to open-label LDX.

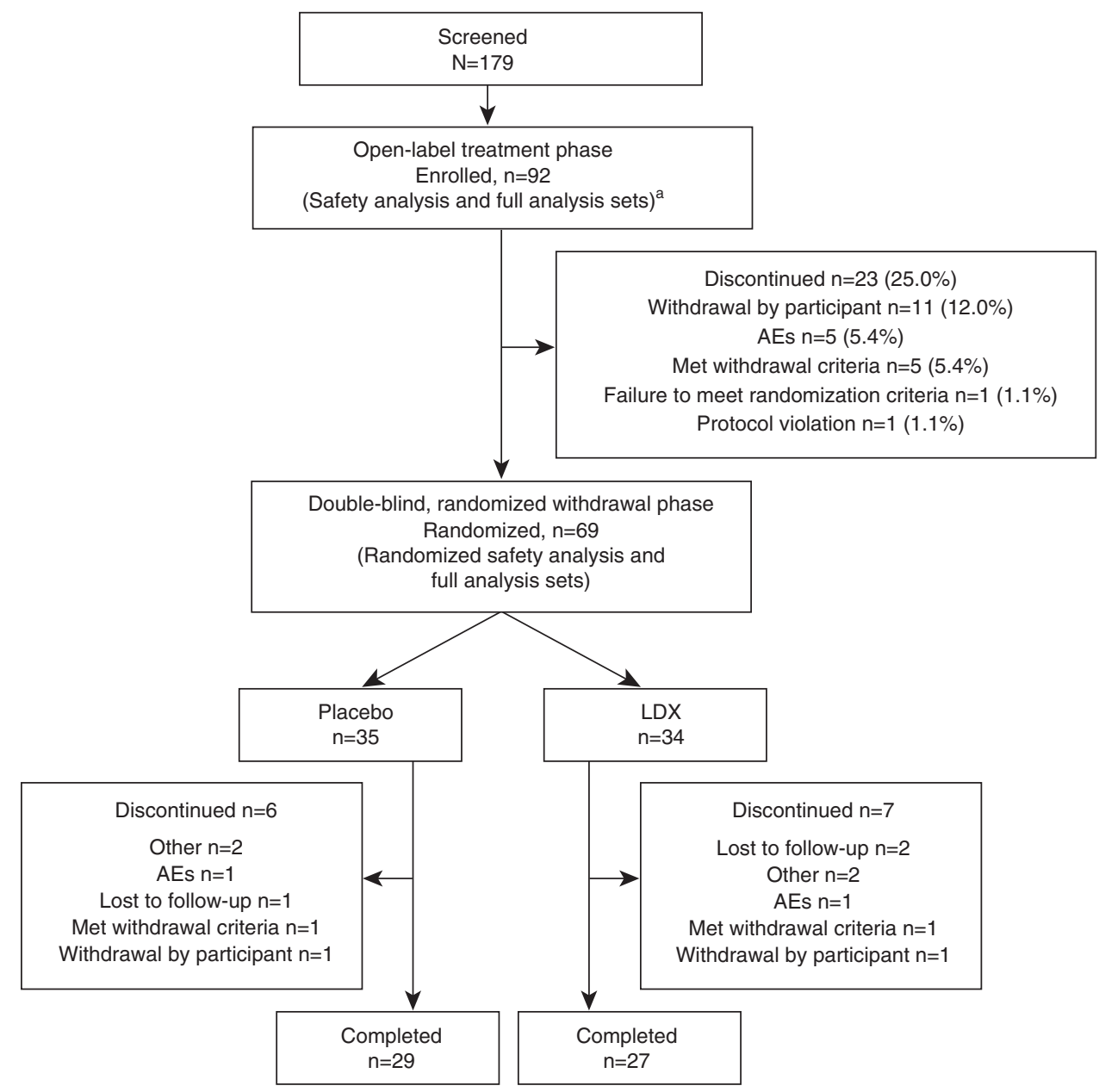

Figure I Participant disposition. a'Safety analysis and full analysis sets included all enrolled participants who took $\geqslant 1$ dose of LDX and had one postbaseline safety/efficacy assessment in the open-label phase. AEs, adverse events; LDX, lisdexamfetamine dimesylate. 
Table I Demographic and Baseline Characteristics

Characteristics

\section{Safety analysis set $(\mathrm{N}=92)$}

$\mathbf{N}$

Sex

Male

Female

Race

White

Non-white

Ethnicity

Hispanic or Latino

Not Hispanic or Latino

Current antipsychotic medication

Risperidone

Quetiapine

Aripiprazole

Olanzapine

Paliperidone
Living situation
Living alone
With family/friend
In a group home

$62 \quad 67$.

30

$\begin{array}{rr}30 & 32.6 \\ 21 & 23.9 \\ 13 & 14.1 \\ 19 & 19.6 \\ 9 & 9.8\end{array}$

\section{II}

49

32

Education

Some high school

High school graduate

Some college

College graduate
$\%$

67.4
32.6

33.7

66.3

|4.1

85.9

32.6

23.9

14. 1

9.8

12.0

53.3

34.8

Randomized safety analysis set $(n=69)$

\begin{tabular}{|c|c|c|c|}
\hline \multicolumn{2}{|c|}{ Placebo $(n=35)$} & \multicolumn{2}{|c|}{$\operatorname{LDX}(n=34)$} \\
\hline$n$ & $\%$ & $n$ & $\%$ \\
\hline 26 & 74.3 & 23 & 67.6 \\
\hline 9 & 25.7 & 11 & 32.4 \\
\hline
\end{tabular}

$\begin{array}{llll}11 & 31.4 & 14 & 41.2 \\ 24 & 68.6 & 20 & 58.8\end{array}$

$\begin{array}{rrrr}5 & 14.3 & 6 & 17.6 \\ 30 & 85.7 & 28 & 82.4\end{array}$

\begin{tabular}{|c|c|c|c|c|c|c|}
\hline & Mean & SD & Mean & SD & Mean & SD \\
\hline Weight (kg) & 87.47 & 16.132 & 85.81 & | 5.794 & 87.71 & | $8.57 \mid$ \\
\hline Years since onset of first schizophrenia episode & 19.14 & 9.716 & & & & \\
\hline Prior hospitalizations & 4.7 & 5.49 & & & & \\
\hline
\end{tabular}

Abreviation: SANS, Scale for the Assessment of Negative Symptoms.

Baseline and demographic characteristics during openlabel and randomized-withdrawal phases are shown in Table 1. These characteristics were similar between the placebo and LDX treatment groups in the randomizedwithdrawal phase. At the start of open-label maintenance, $2.9,15.7,17.1,10.0,22.9$, and $32.9 \%$ of participants received $20,30,40,50,60$, and $70 \mathrm{mg} /$ day LDX, respectively. One participant was unblinded during the study (to medical staff only) after exacerbation of schizophrenia requiring hospitalization during weeks 12-13. Median dose of concurrent atypical antipsychotic medication was 3.0, 350.0, 15.0, 20.0, and $6.0 \mathrm{mg} /$ day for participants receiving risperidone, quetiapine, aripiprazole, olanzapine, and paliperidone, respectively. 


\section{SANS}

During open-label LDX, significant reductions from baseline in SANS-18 total scores were observed from weeks 1-10 (Figure 2a; $P<0.0001$; LOCF). At week 10, participants demonstrated a mean (SD; 95\% CI) change from baseline in SANS-18 scores of $-12.9 \quad(10.04 ; \quad-15.0,-10.8)$ $(P<0.0001$; LOCF; primary endpoint). An alternative post hoc statistical analysis (ie, MMRM) of change from baseline at week 10 demonstrated a LS mean (95\% CI) of -14.5 ( $-16.70,-12.22 ; P<0.0001)$. Comparison of mean difference $(95 \% \mathrm{CI})$ in change from baseline for high- $v s$ low-enrolling sites $(0.44[-4.395,5.265])$ revealed no significant difference $(P=0.8585)$. During the doubleblind/randomized-withdrawal phase, no significant differences in change from weeks 10-14/ET were seen between placebo and LDX groups in the randomized FAS (Figure 2b); similar results were seen in the RES.

The proportion of responders $(\geqslant 20 \%$ reduction in SANS18 total score) generally increased throughout the openlabel phase with $52.9 \%$ responders at week 10 . The proportion of responders during the double-blind/ randomized-withdrawal phase was similar between the placebo and LDX groups in the randomized FAS and RES $(P=\mathrm{NS})$.
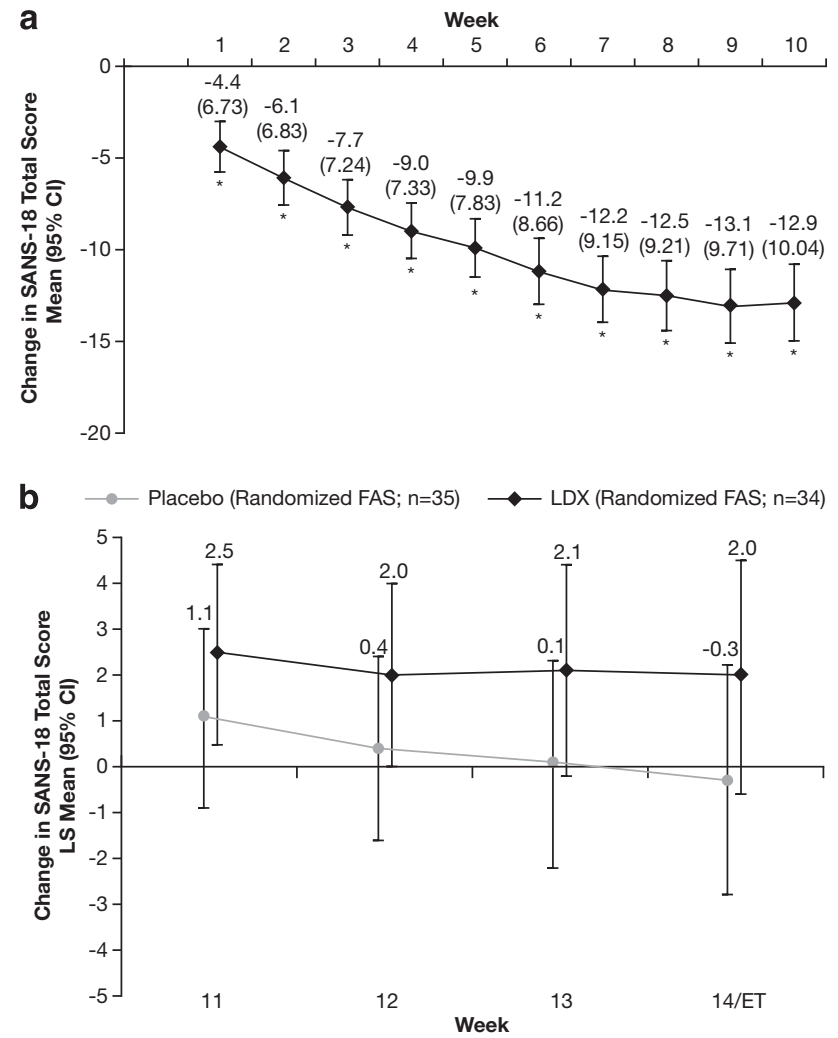

Figure 2 (a) Mean $(95 \% \mathrm{Cl})$ change from baseline in Scale for the Assessment of Negative Symptoms (SANS-18) total score: open-label phase (last observation carried forward; $N=92$ ). Data labels represent mean (SD). $* P<0.000$ I vs baseline. (b) LS mean (95\% Cl) change from randomization baseline (week I0) in SANS-I8 total score: double-blind/ randomized-withdrawal phase (randomized FAS; terminal observation carried forward; $n=69$ ). Data labels represent mean. $P \geqslant 0.2086$ placebo vs LDX for all. ET, end of treatment.
At week 10, participants demonstrated a mean (SD; 95\% CI) change from baseline in SANS-25 scores of -17.0 $(13.31 ;-19.8,-14.3)(P<0.0001 ;$ LOCF $)$. Placebo and LDX groups did not demonstrate significant differences in change from randomization baseline SANS-25 score at any week during the double-blind/randomized-withdrawal phase $(P=$ NS for FAS and RES for weeks 11-14).

\section{PANSS}

Participants also demonstrated statistically significant decreases in PANSS total and all three subscale scores during open-label treatment (weeks $0-10 ; P<0.0001$ for all; LOCF; Figure 3a). No significant differences were observed between placebo and LDX groups in PANSS total and subscale score changes from weeks $10-14 / \mathrm{ET}$ in the randomized FAS during the double-blind/randomizedwithdrawal phase (Figure 3b); similar results were seen in the RES.

\section{Depressive Symptoms}

At week 10, open-label LDX treatment was associated with a minimal mean (SD) CDSS total score change from baseline of -0.7 (2.11). At week 14/ET, mean (SD) CDSS total score changes from week 0 were $-0.7(2.30)$ and $-0.1(2.34)$ for those receiving placebo and LDX, respectively.

\section{Extrapyramidal Symptoms}

LDX treatment was associated with minimal-to-no changes in EPS as assessed by the SAS, BAS, and AIMS.

\section{Adverse Events}

During open-label treatment, 56 participants (60.9\%) reported TEAEs. During the double-blind/randomizedwithdrawal phase, new or worsening TEAEs were reported by 7 participants $(20.0 \%)$ receiving placebo and 11 (32.4\%) receiving LDX. Table 2 summarizes TEAEs with a prevalence $\geqslant 5 \%$ in either phase. In the open-label phase, 38.0 and $19.6 \%$ of 92 participants experienced TEAEs that were considered mild and moderate in intensity, respectively. During the double-blind/randomized-withdrawal phase, 14.3 and $2.9 \%$ of 35 participants receiving placebo had mild and moderate TEAEs; 23.5 and $5.9 \%$ of 34 participants receiving LDX had mild and moderate TEAEs, respectively. Five severe TEAEs were reported by 3 of 92 (3.3\%) participants during the open-label phase: eye swelling, eye pain, erythematous rash $(n=1)$, decreased weight $(n=1)$, and trismus $(n=1)$. During the doubleblind/randomized-withdrawal phase, one participant (2.9\%) in each treatment group experienced a severe TEAE (ie, exacerbation of schizophrenia).

Six participants experienced serious AEs during the trial. During the open-label phase, three participants reported serious TEAEs: chest pain $(n=1)$, schizophrenia exacerbation $(n=1)$, and hallucination and major depression $(n=1)$. During the double-blind/randomized-withdrawal phase, serious TEAEs were reported by two participants receiving placebo (dyspepsia and exacerbation of schizophrenia each reported by one participant) and one 

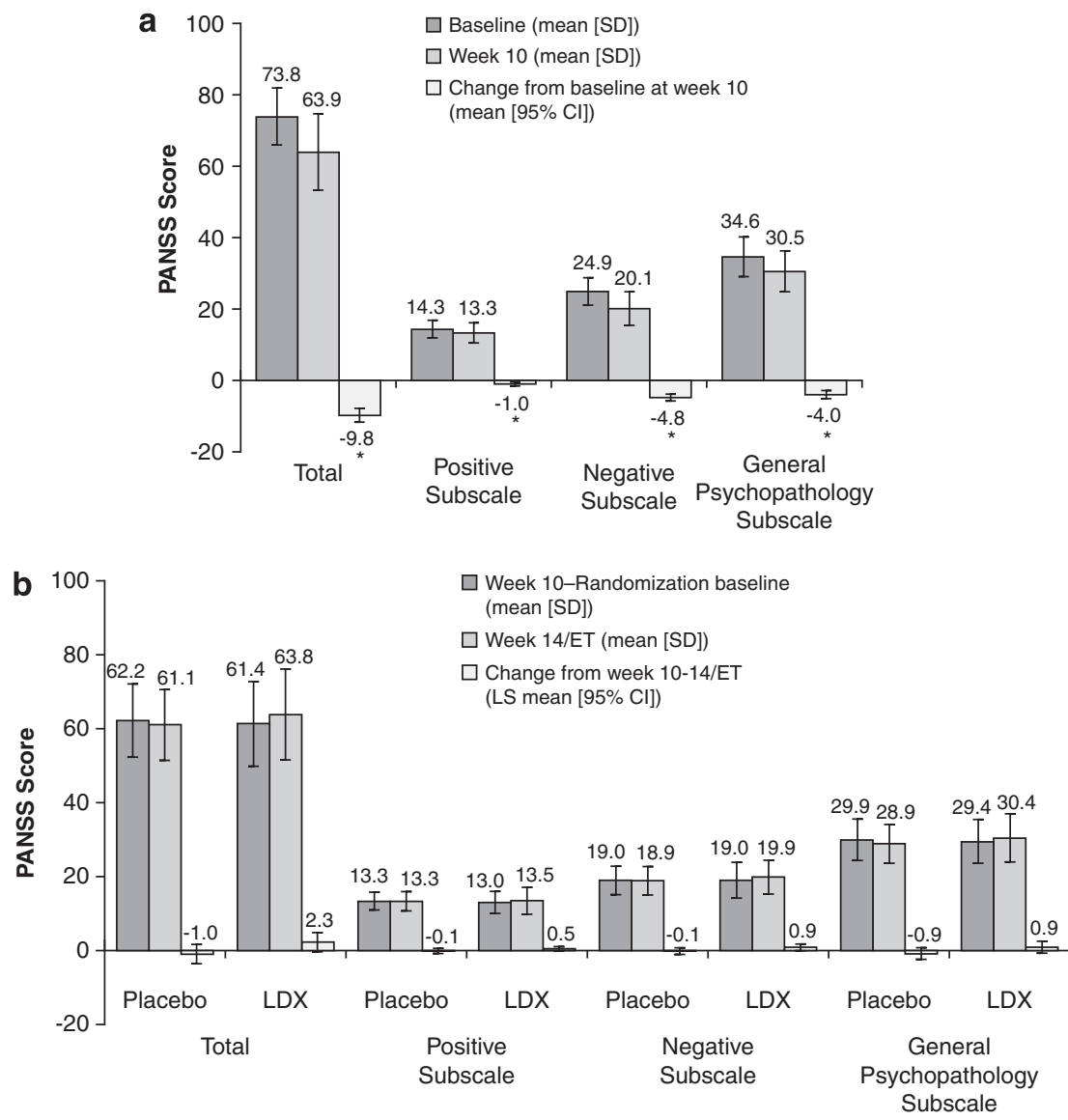

Figure 3 (a) Positive and Negative Syndrome Scale (PANSS) total and subscale scores at baseline and open-label endpoint (week 10 using a last observation carried forward approach; $N=92$ ). Data labels represent mean. $* P<0.000$ I vs baseline. (b) PANSS total and subscale scores at week 10 randomization baseline and week I4/ET of the double-blind/randomized-withdrawal phase (randomized FAS; using a terminal observation carried forward approach; placebo, $n=35$; LDX, $n=34$ ). Data labels represent mean or $L S$ mean values as appropriate for each bar. P $\geqslant 0.0680$ placebo vs $L D X$ for all. ET, end of treatment.

participant receiving LDX (exacerbation of schizophrenia). Overall, seven participants discontinued the study owing to TEAEs. During the open-label phase, five participants had seven TEAEs that led to discontinuation: elevated BP $(n=1)$, exacerbation of schizophrenia $(n=1)$, increased hallucinations $(n=1)$, depression $(n=1)$, major depression $(n=1)$, trismus $(n=1)$, and somnolence $(n=1)$. During the double-blind/randomized-withdrawal phase, two participants (one in each treatment group) discontinued as a result of exacerbation of schizophrenia.

\section{Vital Signs and ECGs}

Open-label treatment with LDX was associated with small mean increases in BP and pulse. At week 10, the mean (SD) changes from baseline in SBP, DBP, and pulse were 2.6 (8.13) $\mathrm{mm} \mathrm{Hg}, \quad 2.3$ (7.12) $\mathrm{mm} \mathrm{Hg}$, and 5.1 (11.74) bpm, respectively. As assessed by ECG, participants demonstrated a mean (SD) increase in heart rate of $4.5(12.8) \mathrm{bpm}$ from baseline at week 10. Mean changes in QT interval, as corrected by Bazett and Fridericia formulas, were 3.9 (14.80) and $0.0(10.92) \mathrm{ms}$, respectively. Participants demonstrated a mean (SD) decrease in body weight of $0.46(4.21) \mathrm{kg}$ from baseline at week 10 .

\section{Amphetamine Withdrawal}

Mean (SD) ACSA total scores were 13.7 (12.00) and 11.7 (11.28) at open-label baseline and mean (SD) changes from open-label baseline at week 14 were -3.8 (9.70) and -1.6 (9.73) for the randomized safety analysis set placebo and LDX groups, respectively. For placebo and LDX groups, respectively, mean (SD) ACSA anxiety and mood subscale scores were 10.0 (9.34) and 8.3 (8.87) at open-label baseline, and changes at week 14 were $-3.0(7.21)$ and $-1.0(8.58)$; mean (SD) ACSA fatigue subscale scores were 3.5 (2.97) and $3.2(2.55)$ at open-label baseline, and changes at week 14 were -1.0 (3.18) and -0.4 (2.65); mean (SD) ACSA craving subscale scores were $0.2(0.76)$ and $0.2(0.99)$ at open-label baseline, and changes at week 14 were $0.2(1.14)$ and $-0.2(1.00)$.

\section{DISCUSSION}

This study represents the first systematic examination of the therapeutic potential of prolonged NSS treatment with amphetamine among outpatients with schizophrenia stabilized on atypical antipsychotic therapy. Our findings suggest negative symptoms can be safely reduced with 
Table 2 Treatment-Emergent Adverse Events With Incidence $\geqslant 5 \%$ During the Open-Label and Double-Blind/Randomized-Withdrawal Phases $^{\text {a }}$

TEAE (MedDRA II.I preferred term)
Safety analysis set $(n=92)$

\begin{tabular}{lcc}
\hline Any TEAE during open-label phase & 56 & 60.9 \\
Headache & 13 & 14.1 \\
Insomnia & 10 & 10.9 \\
Decreased appetite & 10 & 10.9 \\
Dizziness & 8 & 8.7 \\
Dry mouth & 6 & 6.5 \\
Diarrhea & 5 & 5.4 \\
\hline
\end{tabular}

Randomized safety analysis set $(n=69)$

\begin{tabular}{|c|c|c|c|c|}
\hline & \multirow{2}{*}{\multicolumn{2}{|c|}{ Placebo $(n=35)$}} & & \\
\hline & & & \multicolumn{2}{|c|}{$\operatorname{LDX}(n=34)$} \\
\hline & $n$ & $\%$ & $n$ & $\%$ \\
\hline Headache & 2 & 5.7 & 2 & 5.9 \\
\hline Fatigue & I & 2.9 & 2 & 5.9 \\
\hline
\end{tabular}

Abbreviations: MedDRA, Medical Dictionary for Regulatory Activities; TEAEs, treatment-emergent adverse events.

${ }^{a}$ TEAEs during the double-blind/randomized-withdrawal phase represent those events that started or deteriorated on or after the first day of double-blind treatment and no later than 3 days following the last dose of study medication.

adjunctive LDX therapy as long as positive symptoms are stable with antipsychotic therapy. LDX was associated with significant improvements in negative symptoms; $53 \%$ of participants exhibited $20 \%$ or greater decrease in NSS without worsening of positive symptoms.

LDX effects on NSS do not appear to be mediated by improvement in depressive symptoms or EPS, both of which were low at baseline; no meaningful changes were seen throughout the study. These findings suggest that effects of LDX on NSS were likely direct and specific, rather than through improvement of associated symptoms or secondary negative symptoms. Although positive symptoms were slightly improved with open-label LDX, the greater magnitude of improvement in negative symptoms observed suggests that effects of LDX may be predominantly on NSS. A recent post hoc PANSS factors analysis (Dirks et al, 2012), based on an analysis by Marder et al (1997), demonstrated that LDX effects on negative factor analysis paralleled LDX effects on SANS-18 scores, thus providing additional support for effects on NSS. In addition, participants with previous diagnosis of ADHD were excluded. Attention items were excluded from the primary efficacy measure, the SANS-18 score, to ensure that observed effects on NSS were not primarily the result of improved attention.

There were no significant amphetamine withdrawal symptoms noted after LDX discontinuation. Amphetamine withdrawal has not been well studied; however, animal studies suggest behavioral manifestations become prominent within the first 2 days after discontinuation and can persist up to 4 weeks (Paulson et al, 1991). No meaningful differences in response between placebo and LDX were observed on key efficacy measures during the double-blind/ randomized-withdrawal phase. However, 4 weeks might not be long enough for assessment of LDX offset. It is also possible that LDX dose was not fully optimized for some participants during the open-label phase. The decision to limit LDX dose to $70 \mathrm{mg} /$ day was based on approved and effective dosing and established safety profile of LDX for ADHD (Vyvanse package insert, 2012). Future trials may explore additional dosing options in patients with schizophrenia.

The safety profile of LDX throughout the study was largely consistent with that observed in a prior LDX clinical trial in adults with ADHD (Adler et al, 2008). Furthermore, lack of potential withdrawal or rebound symptoms following abrupt LDX discontinuation is consistent with data from the ADHD population (Brams et al, 2011). Overall, 3 of 92 participants experienced exacerbation of schizophrenia; 1 participant reported hallucinations and depression. These results are consistent with a relapse rate of $\sim 15 \%$ annually among patients maintained on atypical antipsychotics (Leucht et al, 2003), and support the notion that, in carefully selected and stable patients, amphetamines may not have psychotogenic effects.

Limiting enrollment to participants with stable illness may reduce risk for exacerbation of schizophrenia and increase in positive symptoms. A previous systematic review suggested individuals with schizophrenia were less likely to exhibit increased psychotic symptoms following psychostimulants if symptoms were in remission $v s$ active psychosis (28.3 vs 51.4\%) (Curran et al, 2004). Such clinical distinctions may be related to neurochemical differences associated with the phase of illness. Assessment of 
$\mathrm{D}_{2}$-receptor occupancy following amphetamine challenge demonstrated similar findings between controls and participants with schizophrenia in remission (Laruelle et al, 1999). Another difference from prior psychostimulant studies was use of the prodrug LDX instead of an immediate-release amphetamine formulation. LDX has demonstrated low interpatient variability in pharmacokinetic variables in healthy adults (Ermer et al, 2010). Preliminary data also suggest that striatal dopamine release exhibits a delayed peak and more prolonged time course with LDX $v s$ immediate-release d-amphetamine in rodent models (Rowley et al, 2011). The equivalent dose of d-amphetamine in the present study (5.9-20.8 mg) also differs from the doses investigated in prior studies (most studies reported doses of $10-40 \mathrm{mg} / \mathrm{day}$, isolated studies up to $100 \mathrm{mg} /$ day) (Chiarello and Cole, 1987). The effects of LDX were based on a 14-week study, which may have limited the risk exposure; longer studies will be needed to ascertain long-term safety of amphetamines in this patient population.

The design of the present study has a number of strengths. The prospective screening phase ensured disease-state stability, the presence of enduring negative symptoms, and minimal or mild positive symptoms as measured by PANSS prospectively and established by retrospective review. In addition, the results should be viewed in light of several limitations. Despite being blinded to the study protocol and details of study progress, as well as the status of an individual participant's progress through the study, the potential for bias among raters cannot be excluded during the open-label phase. Similarly, expectation bias/placebo effects by participants may have contributed to observed treatment effects during the open-label phase. Although the current study design cannot eliminate this possibility, the extended screening phase, the relative length of the open-label phase (10 weeks), and the observation that efficacy increased over time provide some evidence to support the contention that the observed response is related to LDX therapy and not to rater bias. No formal power calculation was performed for the primary efficacy analysis. The limited sample size, especially in the double-blind/randomized-withdrawal phase, may have affected study results. The results may have been further influenced by inclusion of partial responders in the doubleblind/randomized-withdrawal phase. The relatively short randomized-withdrawal (4 weeks) may have contributed to the lack of separation between treatment groups. It is possible that a longer time is needed to observe the worsening of negative symptoms following LDX withdrawal. In addition, based on consideration of the number of participants enrolled in the randomized-withdrawal phase and expected differences, it is not possible to rule out the potential for false-negative findings.

The present results challenge earlier beliefs that patients with schizophrenia should not be exposed to psychostimulants. In carefully selected patients with clinically stable schizophrenia and enduring negative symptoms, adjunctive LDX treatment appeared to be effective in reducing negative symptoms without notable worsening of positive symptoms when given in conjunction with atypical antipsychotic agents. Confirmation of these results with larger, doubleblind, and placebo-controlled trials is needed.

\section{FUNDING AND DISCLOSURE}

Dr Lasser is a former Shire employee and holds stock and/ or stock options in Shire. Dr Dirks is a Shire employee and holds stock and/or stock options in Shire. Dr Nasrallah has received research support and/or consultant fees from Alkermes, AstraZeneca, Forest, Janssen, Merck, Novartis, Otsuka, Pfizer, Sunovion, and Shire. Ms Kirsch is a Shire employee and holds stock and/or stock options in Shire. Dr Gao is a Shire employee and holds stock and/or stock options in Shire. Dr Pucci is an employee of SCI Scientific Communications and Information. SCI was funded by Shire Development LLC for support in writing and editing this manuscript. Dr Knesevich is/has been a consultant to the following companies: AstraZeneca, BioLineRx, Cephalon, Danone Corporation, Forest, Janssen, Johnson \& Johnson, Merck, Novartis, Pfizer, Roche, and Shire; receives/has received grant support from AstraZeneca, BioLineRx, Cephalon, Danone Corporation, Forest Research Institute, Janssen, Johnson \& Johnson, Merck, Novartis, Pfizer, Roche, and Shire. Dr Lindenmayer has received research support and/or consultant fees from Dainippon/Sepracor, Janssen, Lilly, Merck, Otsuka, Roche, and Shire.

\section{ACKNOWLEDGEMENTS}

Clinical research was funded by the sponsor, Shire Development LLC. Editorial assistance in formatting, proofreading, copy editing, and fact checking was provided by SCI Scientific Communications \& Information (SCI). Brian Scheckner and Jann Nielsen, from Shire Development LLC, and Jiannong Wang, a contractor working for Shire Development LLC, also reviewed and edited the manuscript for scientific accuracy. Shire Development LLC provided funding to SCI for support in writing and editing this manuscript. Although the sponsor was involved in the design, collection, analysis, interpretation, and fact checking of information, the content of this manuscript, the ultimate interpretation, and the decision to submit it for publication in Neuropsychopharmacology were made by the authors independently.

\section{REFERENCES}

Addington D, Addington J, Schissel B (1990). A depression rating scale for schizophrenics. Schizophr Res 3: 247-251.

Adler LA, Goodman DW, Kollins SH, Weisler RH, Krishnan S, Zhang Y et al (2008). Double-blind, placebo-controlled study of the efficacy and safety of lisdexamfetamine dimesylate in adults with attention-deficit/hyperactivity disorder. J Clin Psychiatry 69: 1364-1373.

American Psychiatric Association (2000). Diagnostic criteria for schizophrenia. In: Diagnostic and Statistical Manual of Mental Disorders DSM-IV-TR, 4th edn, Text Revision. American Psychiatric Association: Washington, DC, pp 312-313.

Andreasen NC (1984): Scale for the Assessment of Negative Symptoms (SANS). http://www.movementdisorders.org/UserFiles/file/Long_SANS_2000_publish\%281\%29.pdf. Accessed 23 March 2012.

Angrist B, Peselow E, Rubinstein M, Corwin J, Rotrosen J (1982). Partial improvement in negative schizophrenic symptoms after amphetamine. Psychopharmacology (Berl) 78: 128-130. 
Barnes TR (1989). A rating scale for drug-induced akathisia. $\mathrm{Br} J$ Psychiatry 154: 672-676.

Brams M, Weisler R, Findling R, Gasior M, Hamdani M, FerreiraCornwell $\mathrm{C}$ et al (2011). Maintenance of efficacy of lisdexamfetamine dimesylate in adults with attention-deficit/hyperactivity disorder: randomized withdrawal design. Poster presented at American Psychiatric Association's 164th Annual Meeting. 1418 May 2011; Honolulu, HI.

Buckley PF, Stahl SM (2007). Pharmacological treatment of negative symptoms of schizophrenia: therapeutic opportunity or cul-de-sac? Acta Psychiatr Scand 115: 93-100.

Cesarec Z, Nyman AK (1985). Differential response to amphetamine in schizophrenia. Acta Psychiatr Scand 71: 523-538.

Chiarello RJ, Cole JO (1987). The use of psychostimulants in general psychiatry. A reconsideration. Arch Gen Psychiatry 44: 286-295.

Curran C, Byrappa N, McBride A (2004). Stimulant psychosis: systematic review. Br J Psychiatry 185: 196-204.

Desai NG, Gangadhar BN, Pradhan N, Channabasavanna SM (1984). Treatment of negative schizophrenia with d-amphetamine. Am J Psychiatry 141: 723-724.

Dirks B, Lasser R, Nasrallah H, Kirsch C, Adeyi B, Scheckner B et al (2012). Effect of adding lisdexamfetamine dimesylate to antipsychotics on Predominant Negative Symptoms of Schizophrenia: Analysis of PANSS Factors. Poster presented at American Psychiatric Association's 165th Annual Meeting; 5-9 May 2012; Philadelphia, PA.

Ermer J, Homolka R, Martin P, Buckwalter M, Purkayastha J, Roesch B (2010). Lisdexamfetamine dimesylate: linear doseproportionality, low intersubject and intrasubject variability, and safety in an open-label single-dose pharmacokinetic study in healthy adult volunteers. J Clin Pharmacol 50: 1001-1010.

Foussias G, Remington G (2010). Negative symptoms in schizophrenia: avolition and Occam's razor. Schizophr Bull 36: 359-369.

Geodon (package insert) Pfizer: New York, NY; 2010.

Guillin O, Abi-Dargham A, Laruelle M (2007). Neurobiology of dopamine in schizophrenia. Int Rev Neurobiol 78: 1-39.

Guy W (1976). ECDEU Assessment Manual for Psychopharmacology, Revised. Pub. No. (ADM) 76-338, NIMHUS Department of Health, Education and Welfare: Rockville, MD.

Kay SR, Fiszbein A, Opler LA (1987). The Positive and Negative Syndrome Scale (PANSS) for Schizophrenia. Schizophr Bull 13: 261-276.

Kirkpatrick B, Fenton WS, Carpenter WTJ, Marder SR (2006). The NIMH-MATRICS consensus statement on negative symptoms. Schizophr Bull 32: 214-219.

Laruelle M, Abi-Dargham A, Gil R, Kegeles L, Innis R (1999). Increased dopamine transmission in schizophrenia: relationship to illness phases. Biol Psychiatry 46: 56-72.

Laruelle M, Frankle WG, Narendran R, Kegeles LS, Abi-Dargham A (2005). Mechanism of action of antipsychotic drugs: from dopamine $\mathrm{D}(2)$ receptor antagonism to glutamate NMDA facilitation. Clin Ther 27(Suppl A): S16-S24.
Laughren T, Levin R (2011). Food and Drug Administration commentary on methodological issues in negative symptom trials. Schizophr Bull 37: 255-256.

Leucht S, Barnes TR, Kissling W, Engel RR, Correll C, Kane JM (2003). Relapse prevention in schizophrenia with new-generation antipsychotics: a systematic review and exploratory metaanalysis of randomized, controlled trials. Am J Psychiatry 160: $1209-1222$

Lieberman JA, Kane JM, Alvir J (1987). Provocative tests with psychostimulant drugs in schizophrenia. Psychopharmacology (Berl) 91: 415-433.

Marder SR, Davis JM, Chouinard G (1997). The effects of risperidone on the five dimensions of schizophrenia derived by factor analysis: combined results of the North American trials. J Clin Psychiatry 58: 538-546.

McGregor C, Srisurapanont M, Mitchell A, Longo MC, Cahill S, White JM (2008). Psychometric evaluation of the Amphetamine Cessation Symptom Assessment. J Subst Abuse Treat 34: 443-449.

Milev P, Ho BC, Arndt S, Andreasen NC (2005). Predictive values of neurocognition and negative symptoms on functional outcome in schizophrenia: a longitudinal first-episode study with 7-year follow-up. Am J Psychiatry 162: 495-506.

Olie JP, Spina E, Murray S, Yang R (2006). Ziprasidone and amisulpride effectively treat negative symptoms of schizophrenia: results of a 12-week, double-blind study. Int Clin Psychopharmacol 21: 143-151.

Paulson PE, Camp DM, Robinson TE (1991). Time course of transient behavioral depression and persistent behavioral sensitization in relation to regional brain monoamine concentrations during amphetamine withdrawal in rats. Psychopharmacology (Berl) 103: 480-492.

Rowley H, Heal D, Hackett D (2011). Simultaneous measurement with hysteresis analyses of the effects of lisdexamfetamine dimesylate and d-amphetamine on striatal levels of extracellular dopamine, locomotor activity, and plasma drug concentrations in freely-moving rats. Poster presented at: New Clinical Drug Evaluation Unit Annual Meeting; 13-16 June 2011; Boca Raton, FL.

Simpson GM, Angus JW (1970). A rating scale for extrapyramidal side effects. Acta Psychiatr Scand Suppl 212: 11-19.

Stahl SM, Buckley PF (2007). Negative symptoms of schizophrenia: a problem that will not go away. Acta Psychiatr Scand 115: 4-11.

Storosum JG, Elferink AJ, van Zwieten BJ, van Strik R, Hoogendijk WJ, Broekmans AW (2002). Amisulpride: is there a treatment for negative symptoms in schizophrenia patients? Schizophr Bull 28: 193-201.

Tamminga CA (2006). The neurobiology of cognition in schizophrenia. J Clin Psychiatry 67(Suppl 9): 9-13.

Toda M, Abi-Dargham A (2007). Dopamine hypothesis of schizophrenia: making sense of it all. Curr Psychiatry Rep 9: 329-336.

Vyvanse (package insert). Shire US: Wayne, PA; 2012. 